\title{
Rigid Films of an Anionic Porphyrin and a Dialkyl Chain Surfactant
}

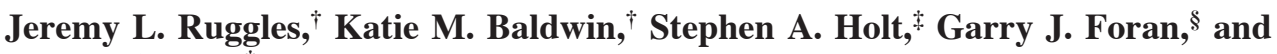 \\ Ian R. Gentle*,†
}

School of Molecular and Microbial Sciences, The University of Queensland, Brisbane, Australia 4072, ISIS Pulsed Neutron \& Muon Source, Rutherford Appleton Laboratory, Chilton, Didcot OX11 OQX, United Kingdom, and Australian Nuclear Science and Technology Organization, PMB 1, Menai, NSW, Australia 2234

Received: November 21, 2006; In Final Form: February 16, 2007

\begin{abstract}
The 2D complex formed at the air-water interface between the dialkyl chain cationic surfactant, dihexadecyldimethylammonium bromide, and the anionic porphyrin, tetrakis-(4-sulfonatophenyl) porphine, was studied using surface pressure-area isotherms as well as X-ray and neutron reflection measurements. The surface structure of these films was determined by the use of simultaneously constrained analysis of the neutron and X-ray reflectometry data and BAM images. Isotopic contrast variation methods were employed to enhance the information content of the neutron reflection data. The rigid complex forms at the interface due to the electrostatic interaction between the cationic headgroups of the surfactant and the anionic functional groups at the meso position of the porphyrin. The surface pressure-area isotherms show three distinct regions on compression: an initial condensed phase that ends with a pressure peak at $36 \mathrm{mN} \mathrm{m}^{-1}$, a second plateau region of high compressibility, and a final condensed phase. BAM images show that at the beginning of the plateau region in the isotherm there is complete surface coverage by a monolayer. The constrained simultaneous fitting of neutron and X-ray data measured just prior to and after the pressure peak shows a structurally similar 2D complex at the interface. Modeling of X-ray reflectometry data also reveals that in the final highpressure phase the film has folded to form a trilayer. The conclusion is that the plateau region of the isotherm is due to the formation of trilayer surface coverage through localized buckling or folding, and that after this is complete there is some condensation before final film collapse.
\end{abstract}

\section{Introduction}

It is of great interest to fabricate films of functional molecules in which the functional component, in this case a porphyrin, is constrained to the plane of the substrate and arranged discretely on the surface such that electron transfer is possible in a controlled way between adjacent molecules. Such methodology is attractive to the surface scientist as it offers the possibility of constructing nanoscale electronic devices, ${ }^{1}$ gas sensors, ${ }^{2}$ and photoconductors. ${ }^{3,4}$ Porphyrins are of interest as functional and structural components for the construction of molecular materials for electronic and optical applications. The central ring of the macrocycle contains delocalized $\pi$ electrons, which are the reason for their characteristic deep colors and confer useful electronic and electro-optical properties. The presence of the conjugated $\pi$ electron ring, however, leads to $\pi-\pi$ associations between molecules and a tendency to aggregate in a random fashion in solution and on surfaces. ${ }^{5,6}$ These aggregates can quench the efficient energy transfer between molecules.

Recent experiments have demonstrated that control over the porphyrin orientation with respect to the interface can be achieved by Coulombic interactions between an insoluble monolayer at the air-water interface, such as a phospholipid monolayer with negatively charged headgroups, and oppositely charged functional groups on the periphery of the porphyrin

* Corresponding author. Telephone: +61 73365 4800. Fax: +617 3365 4299. E-mail: i.gentle@uq.edu.au.

$\dagger$ The University of Queensland.

$\doteqdot$ ISIS, Rutherford Appleton Laboratory.

$\S$ Australian Nuclear Science and Technology Organization. molecules. ${ }^{7-9}$ These systems can be transferred to suitable substrates using either Langmuir-Blodgett (LB) or LangmuirSchaeffer (LS) techniques, while maintaining the molecular arrangement. Researchers were able to demonstrate that the anionic phospholipid-cationic porphyrin system: dimyristoyl phosphatidic acid (DMPA) and tetrakis-(4-methylpyridyl)porphyrin (TMPyP), could be transferred to substrates using LB techniques, and that some structure had been retained from the surface organization. ${ }^{8-10}$

In this work, the overlayer at the air-water interface was formed by a cationic surfactant with twin alkyl tails, ${ }^{11,12}$ dihexadecyldimethylammonium bromide (DHDABr). Such alkyl quaternary ammonium bromides demonstrate surface characteristics similar to those of the anionic phospholipids, that is, formation of a stable surface monolayer, a bulky charged headgroup, and twin tails composed of saturated alkyl chains. ${ }^{13}$ The porphyrin is the water-soluble anionic tetrakis-(4-sulfonatophenyl)porphyrin (TSPP, see Figure 1). There has been interest in similar systems recently due to the unusual surface pressure isotherm resulting from a complex formed between the analogous $\mathrm{C}_{18}$ alkylammonium bromide surfactant and the TSPP porphyrin. ${ }^{14,15}$ On compression, the isotherm exhibits a pressure peak followed by a plateau region of high stability, which offers the possibility of film transfer while maintaining the surface structure. The researchers postulated that this region represented the collapse of the monolayer into a trilayer structure at the interface; however, until now no direct characterization of these complex systems has been presented. In this paper we report the application of a combination of X-ray and neutron reflectometry to gain detailed information of the structure of 


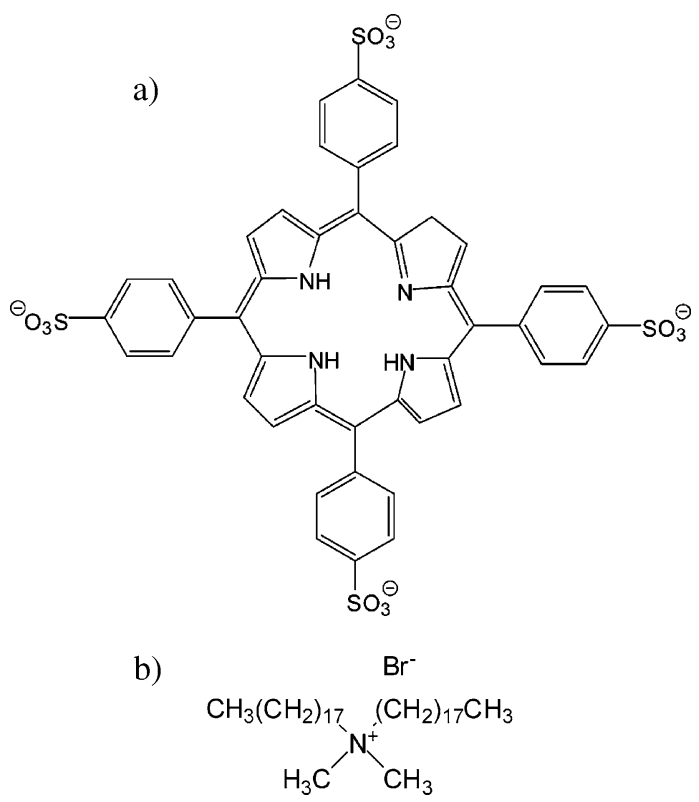

Figure 1. Molecules used in monolayer experiments; tetrasulfonatophenylporphyrin, TSPP, and dihexadecyldimethylammonium bromide, DHDABr. The deuterated version of DHDABr has the hydrogen atoms on the alkyl tails substituted by deuterium atoms, but the headgroup methyl groups remain protonated.

surfactant-porphyrin complexes at the air- water interface as a function of surface pressure.

\section{Materials and Methods}

Dihexadecyldimethylammonium bromide (DHDABr) was obtained from Aldrich and used as received. Tetrakis-(4sulfonatophenyl)porphyrin (TSPP) was supplied by Frontier Scientific and used without further purification. The solvents were obtained from Aldrich (Aldrich, A.C.S. spectrophotometric grade $99.8 \%$ ) and used as received. The chain-deuterated surfactant, $d_{66}$-dihexadecyldimethylammonium bromide, was obtained from the laboratory of Prof. R. K. Thomas, University of Oxford, in a crystalline form and used as received. A mixture of dichloromethane and methanol in a $3: 1 \mathrm{v} / \mathrm{v}$ ratio was used as the spreading solvent.

The monolayer films were established by co-spreading a mixture of the surfactant (DHDABr) and the porphyrin (TSPP). The $\pi-A$ isotherms were measured using a polytetrafluoroethylene trough (NIMA Technologies, Coventry, U.K.), with Wilhelmy plates made from Whatman Chr 1 chromatography paper. For all measurements, films were spread on a subphase of Milli-Q water (18.2 $\mathrm{m} \Omega$ resistivity) at $21.5 \pm 1{ }^{\circ} \mathrm{C}$.

Neutron reflectometry experiments were performed on the time-of-flight SURF reflectometer at the ISIS Spallation Neutron Source, Rutherford Appleton Laboratory, Didcot, U.K. ${ }^{16}$ Measurements were performed at incident angles of $1.5^{\circ}$ and $0.8^{\circ}$, and the reflectivity of a $\mathrm{D}_{2} \mathrm{O}$ subphase was used to normalize the profiles. Data were collected for momentum transfer $Q_{z}=$ $(4 \pi / \lambda) \sin \theta$ between 0.026 and $0.612 \AA^{-1}$, although typically the incoherent background scattering becomes dominant for values of $Q_{z}$ greater than $0.3 \AA^{-1}$. Surface pressure-area measurements were recorded at the same time as the neutron reflectometry profiles. Isotopic substitution was utilized to enhance the information content of neutron scattering experiments based on the large difference in scattering length between ${ }^{1} \mathrm{H}$ and ${ }^{2} \mathrm{D} .{ }^{17} \mathrm{~A}$ combination of deuterated and protonated surfactants and subphase materials $\left(\mathrm{D}_{2} \mathrm{O}\right.$ and air-contrast matched water (ACMW)) were used at each surface pressure.
For all reflectometry measurements, the films were allowed to stand for $15 \mathrm{~min}$, compressed to the desired pressure, and then allowed to equilibrate for a further $15 \mathrm{~min}$ before collection of data.

X-ray reflectometry measurements were carried out on the undulator beam line, BL-15-IDC (operated by ChemMatCARS) at the Advanced Photon Source (APS, Argonne National Laboratory, IL). The wavelength of the radiation was $1.5 \AA$, and the incident X-ray beam had a vertical size at the sample of $0.1 \mathrm{~mm}$. At the APS, the $\pi-A$ isotherms and reflectivity profiles were recorded on films spread on subphases in a custommade PTFE trough. The trough had been fitted with a pressure sensor and software from NIMA Technologies, Coventry, U.K., using Wilhelmy plates made from Whatman Chr 1 chromatography paper. Reflectometry data were collected for all the films to $0.70 \AA^{-1}$ in $Q_{z}$.

Reflectivity profiles were fitted using the Motofit program ${ }^{18}$ recently developed at ANSTO, Australia. The interface is divided into a series of slabs or layers using three parameters, layer thickness, $d$ (in units of $\AA$ ), scattering length density, SLD $\left(\times 10^{-6} \AA^{-2}\right)$ represented as $\rho\left(\times 10^{-6} \AA^{-2}\right)$ for X-rays and $N_{b}$ $\left(\times 10^{-6} \AA^{-2}\right)$ for neutrons, and a Gaussian roughness between layers. Known values were used for model-independent data such as scattering length density of subphase and air, and a fixed interface roughness of $3 \AA$ was used. The data were modeled using combinations of data sets representing the isotopically contrasting components of the same system with constraints imposed on the common thicknesses and roughnesses of the system. The simplest system, that is, the least number of separate boxes necessary to describe the system was used, based on the lowest $\chi^{2}$ from fitting measured data.

Brewster angle microscopy (BAM) was performed in the Nima trough using a BAM2 instrument from NFT (Göttingen, Germany). This technique is sensitive to the surface density and to the anisotropy of phase domains in monolayers, where the reflectivity of a planar interface between two media depends on the polarization of the incident light and on the angle of incidence. For a Fresnel interface (an interface where the refractive index changes steeply) and for light polarized with the electric field in the plane of incidence, the reflectivity vanishes at the Brewster angle $\left(53.15^{\circ}\right.$ for the air-water interface). Images were recorded throughout the entire range of the surface pressure-area isotherm. There were some difficulties in obtaining images at lower surface pressures due to the rapid movement of structures across the surface, which caused slightly blurred images.

\section{Results}

$\pi-\boldsymbol{A}$ Isotherms. DHDABr forms an insoluble monolayer when spread at the air-water interface. Figure 2 shows surface pressure-area isotherms of monolayers of the surfactant DHDABr alone (dots), and as a 4:1 molar mixed monolayer film with the porphyrin TSPP at the air-water interface (solid line). Isotherms are expressed as area per DHDABr molecule; thus any expansion with respect to the pure surfactant is due to the presence of the porphyrin at the interface. The porphyrin, TSPP, is soluble in water, and hence no isotherm can be measured for TSPP alone. These results are similar to those observed by other workers. ${ }^{19}$

The pure surfactant isotherm shows liftoff occurring at a molecular area of $170 \AA$. $^{2}$ From liftoff through to $\sim 110 \AA^{2}$ the isotherm shows a region of high compressibility associated with a mixture of liquid expanded and gaseous states at low surface pressures. The gradual increase in surface pressure is attributed 


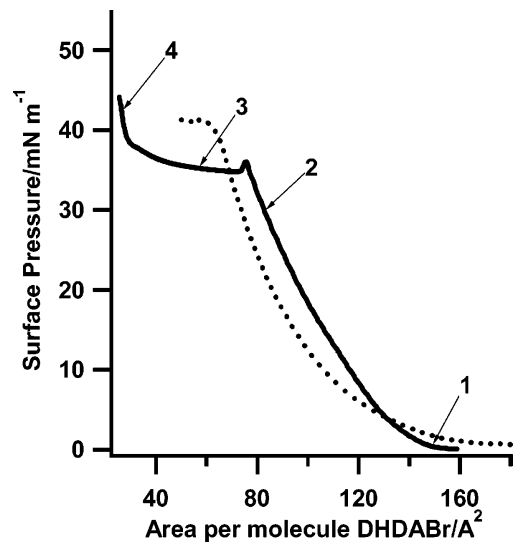

Figure 2. Surface pressure-area isotherms of monolayers films of DHDABr (dots) and a 4:1 molar mixture of DHDABr-TSPP (solid line). The numbered parts of the isotherm refer to the stages of film compression when BAM images were recorded, and for 2-4 for when reflectometry data was measured.

almost solely to interaction of the tails in the DHDABr. With increasing surface pressure, the film forms a liquid condensed state as the pressure rises approximately linearly to a collapse pressure of $42 \mathrm{mN} \mathrm{m}^{-1} \cdot{ }^{11}$ Extrapolation of the linear region of the isotherm to zero pressure indicates a molecular area of 100 $\AA^{2}{ }^{12,20}$ This is considerably larger than twice the molecular area of the single-chained analogue $\mathrm{C}_{16} \mathrm{TABr}$ of $44 \AA^{2} /$ molecule ${ }^{21}$ measured as a surface excess film rather than a compressed monolayer. The presence of the twin alkyl tails inhibits the formation of a close-packed arrangement of headgroups (see Figure 5).

DHDABr and TSPP form a mixed monolayer (we will use this term although the layer is clearly more complex than a single layer of molecules) when co-spread from solution at the air-water interface. There is Coulombic coordination between the positively charged headgroup of the surfactant and the negatively charged sulfonato groups located at the meso position of the porphyrin. Several molar mixtures of the surfactantporphyrin films were examined: a 4:1 charge-balanced mixture, a 1:1 mixture with excess porphyrin, and an 8:1 film with an excess of surfactant. All three mixtures resulted in isotherms similar to that shown in Figure 2, indicating formation of a film similar to that for 4:1 in each case. The isotherm is different from that of the surfactant alone and can be divided into three distinct regions as the film is compressed: an initial region with an approximately linear increase in surface pressure, a plateau region in which surface pressure remains nearly constant, and a third region of steadily increasing pressure. The mixed film isotherm also shows a surface pressure peak, which occurs between the first two regions at a surface pressure of $36 \mathrm{mN}$ $\mathrm{m}^{-1}$. There has been speculation in the literature as to the cause of such a peak followed by a plateau region in the surfacepressure-area compression isotherms, with the majority agreeing that it represents the nucleation of folding or buckling of monolayers into trilayers at the interface. ${ }^{19,22-24}$ There is also evidence of the same peak occurring in the isotherms of systems involving hydrogen bonding between guanidinium ions and sulfonato functional groups, ${ }^{25-27}$ which is similar to the combination of sulfonato groups on the porphyrin and the quaternary nitrogen group of the surfactant in this present study.

These mixed monolayer films are extremely stable in the plateau region, which extends from a molecular area per DHDABr molecule of 76 to $29 \AA,^{2}$ showing no loss of surface pressure even after $24 \mathrm{~h} .{ }^{19}$ The system was cycled between low surface pressure and a pressure just below the surface pressure peak (data not shown), and hysteresis was observed in the decompression cycle, which we believe is due to the solubility of the porphyrin. ${ }^{14}$ The subsequent compression is identical to that of the first cycle but at a slightly lower area due to the losses. Similar cycling experiments also showed that the formation of the second condensed phase (position 4 in Figure 2) was an irreversible process.

Reflectometry Measurements of Langmuir Films. X-ray reflectometry measurements were recorded on either side of the surface pressure peak (at 30 and $35 \mathrm{mN} \mathrm{m}^{-1}$ ) and also in the second condensed phase at $42 \mathrm{mN} \mathrm{m}^{-1}$ (represented by arrows 2, 3, and 4 in Figure 2). Complementary neutron reflectometry measurements were performed at the two lower surface pressures on equivalent systems with isotopic variation of the surfactant and/or subphase. Reflectometry provides information about the structure of the monolayer perpendicular to the surface, which enables the determination of layer thicknesses and density within the monolayer.

The neutron data sets involved two different forms of the surfactant DHDABr: one with protonated alkyl chains and the other with the chains fully deuterated. The protonated surfactant was spread on a $\mathrm{D}_{2} \mathrm{O}$ subphase for maximum contrast, and the deuterated surfactant on an ACMW subphase. The large $Q$-range of the X-ray data provides a good determination of the layer thicknesses and is a useful constraint on the neutron data. The isotopic variation of the neutron data sets when refined simultaneously with the X-ray data provides further information on the layer densities and composition and helps remove the ambiguity associated with modeling a single data set. In particular, the use of the ACMW subphase removes subphase scattering, and so for this contrast all information arises from the spread film. Plots of the results from the constrained simultaneous modeling are shown in Figure 3, along with a depth profile of the scattering length density of the layers in the inset. The data are represented as $R \cdot Q_{z}{ }^{4}$ vs $Q_{z}$ as this eliminates the majority of the Fresnel scattering arising from the sharp interface between the air and liquid, and this allows the structural information within the surface layer to be more easily observed.

In order to fit the data, a model was assumed in which the surface was divided into two regions, which we denote the headgroup (lower layer, thickness $t_{\text {hgp }}$ ) and tail (upper layer, thickness $t_{t}$ ) regions. A single-layer model was not able to produce sufficiently good fits to the data. It must be borne in mind that other models may also produce reasonable fits; however, our goal was to find the simplest, chemically reasonable model that produced adequate results. The expected values of the scattering length density of the components of the monolayer within this model were calculated using layer thicknesses obtained by fitting using Motofit, along with the molecular area obtained from the surface pressure-area isotherms and some knowledge of the expected chemistry of individual layers. In order to derive a theoretical model system for comparison with experimental data, we assumed two layers: the lower layer includes the porphyrins, surfactant head groups, and the first methylene carbon of the chains, and the upper layer includes the rest of the chains. ${ }^{20}$ These calculated values, along with the best fits for the reflectometry data, are shown in Table 1.

From the reflectometry plots it can be seen that the profile does not change significantly for the measurements recorded just prior to the pressure peak $\left(30 \mathrm{mN} \mathrm{m}^{-1}\right)$ and just after the pressure peak ( $35 \mathrm{mN} \mathrm{m}^{-1}$, arrows 2 and 3 in Figure 2, and profiles Figure 3, parts a and b). This is most obvious for the 

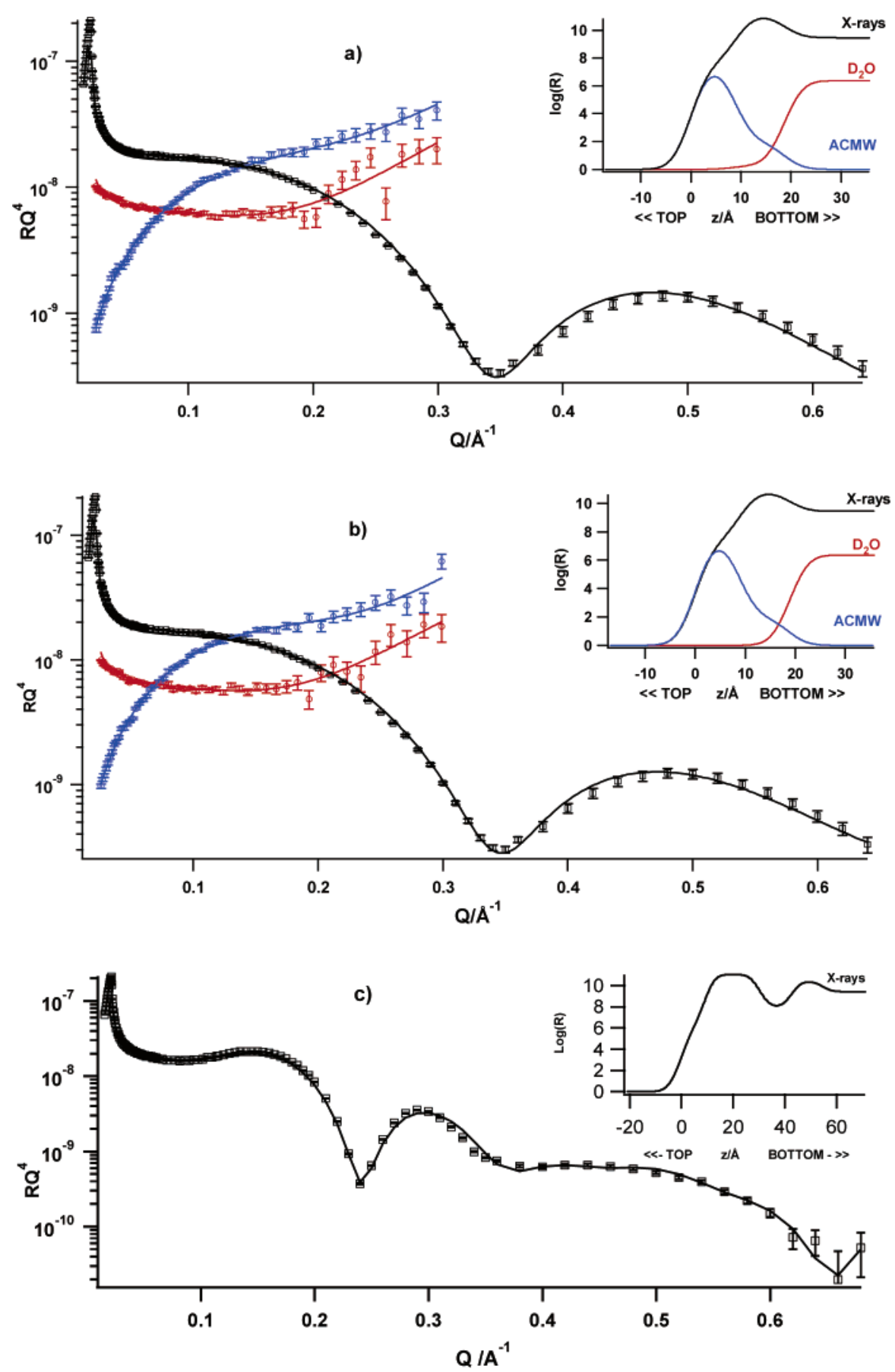

Figure 3. X-ray and neutron reflectometry data for the 4:1 DHDABr-TSPP complex measured at (a) $30 \mathrm{mN} \mathrm{m}^{-1}$, (b) $34.5 \mathrm{mN} \mathrm{m}^{-1}$, and (c) 45 $\mathrm{mN} \mathrm{m}^{-1}$ (arrows 2-4 in Figure 2). Inset shows scattering length density as a function of depth from interface profile corresponding to the fit displayed as a solid line in each reflectometry data set.

X-ray data, which extends out to $0.7 \AA^{-1}$ and over 9 orders of magnitude of reflectivity. The best fit to the data was a twobox model, consisting of a hydrophobic layer nearest the air and a hydrophilic layer adjacent to the subphase. At $30 \mathrm{mN}$ $\mathrm{m}^{-1}$, just before the pressure peak, the two layers are of almost equal thicknesses of 8.9 and $9.7 \AA$ for $t_{t}$ and $t_{h g p}$, respectively. At $35 \mathrm{mN} \mathrm{m}^{-1}$ the two layers have not changed thickness or density to a significant degree; $t_{t}$ is $8.9 \AA$ and $t_{h g p} 10.0 \AA$. The thickness of the tail region is less than expected on the basis of observations of monolayers of alkyl chains of surfactants with single alkyl chains, which when compressed normally become more upright and ordered. This would imply that the upper layer should be nearer to the length expected for a fully extended alkyl chain of 16 carbon atoms $(20.095 \AA)$.
Our modeling of X-ray reflectivity data collected from a DHDABr monolayer spread alone at the interface and compressed to $30 \mathrm{mN} \mathrm{m}^{-1}$ reveals a two-layer system, the details of which are shown in Table 1 . The best fit reveals another two-box model with the upper hydrophobic alkyl chain layer also thinner $\left(t_{t}=11.1 \AA\right)$ than theory would have predicted. The air-water interface roughness for the fit was $4.2 \AA$, larger than the accepted value for a water-air interface of $2.8 \AA$ commonly attributed to capillary surface waves, and it indicates that the diffuse interface between air and tail material was difficult to resolve.

It is possible to use the results of fitting the neutron isotopically contrasted reflectometry data to better understand the composition of the layers of the complex. This is achieved 
TABLE 1: Results of Modeling Reflectometry Data

\begin{tabular}{|c|c|c|c|c|c|c|c|c|c|}
\hline \multirow{3}{*}{$\begin{array}{l}\text { surface pressure } \\
\qquad\left(\mathrm{mN} \mathrm{m}^{-1}\right)\end{array}$} & & & \multicolumn{3}{|c|}{$\begin{array}{c}\text { SLD } \\
\left(\times 10^{-6} \AA^{-2}\right) \\
\end{array}$} & \multirow[b]{3}{*}{$\chi^{2}$} & \multicolumn{3}{|c|}{$\begin{array}{l}\text { calculated SLD } \\
\left(\times 10^{-6} \AA^{-2}\right)\end{array}$} \\
\hline & \multirow{2}{*}{\multicolumn{2}{|c|}{$\begin{array}{l}\text { layer thickness } \\
(\AA)\end{array}$}} & \multicolumn{2}{|c|}{$\mathrm{N}_{\mathrm{b}}$} & \multirow[b]{2}{*}{$\rho$} & & & & \multirow[b]{2}{*}{$\rho$} \\
\hline & & & $h$-DHDABr & $d$-DHDABr & & & $h$-DHDABr & $d$-DHDABr & \\
\hline 30 & $t_{t}$ & 11.1 & 0.07 & 7.06 & 5.01 & 0.03 & -0.41 & 7.87 & 8.73 \\
\hline DHDABr & $t_{h g p}$ & 4.6 & 5.08 & 0.45 & 14.8 & & 0.59 & 1.89 & 7.11 \\
\hline 30 & $t_{t}$ & 8.9 & 0.26 & 6.61 & 7.55 & 0.80 & -0.41 & 7.66 & 8.30 \\
\hline 4:1 complex & $t_{h g p}$ & 9.7 & 1.36 & 3.01 & 11.1 & & 0.82 & 1.29 & 5.25 \\
\hline \multirow{2}{*}{$\begin{array}{l}35 \\
4: 1 \text { complex }\end{array}$} & $t_{t}$ & 8.9 & -0.09 & 7.30 & 7.20 & 0.77 & -0.64 & 9.84 & 11.04 \\
\hline & $t_{h g p}$ & 10.0 & 1.49 & 3.41 & 10.8 & & 1.03 & 1.41 & 6.52 \\
\hline
\end{tabular}

by adjusting the composition of the headgroup and tail layers in our model system such that the calculated values are equivalent to the best fit to the data obtained from fitting. For the neutron contrast of using $d_{66}$-DHDABr with TSPP on ACMW, the only contribution to the SLD comes from the monolayer. At $30 \mathrm{mN} \mathrm{m}^{-1}$, the fitted SLD for the upper layer is lower than the value calculated on the basis of the initial assumption of inclusion of one methylene carbon. Hence, it is necessary to include more tail material, 6.2 methylene carbons per surfactant molecule, in the lower layer to approximate the lower measured density of the upper layer. The other contrast utilizing $h$-DHDABr and TSPP on a $\mathrm{D}_{2} \mathrm{O}$ subphase enables us to calculate the amount of subphase penetration into the upper layer, which in this case is 2 water molecules per porphyrin molecule. This small amount of subphase penetration is appropriate for material which is hydrophobic in nature. Using the same reasoning we can calculate the composition of the lower layer, and after including the extra 6.2 methylene carbons from the tails we require 11.2 water molecules per porphyrin molecule. We used the same reasoning to determine that data recorded at the slightly higher surface pressure in the plateau region required 7.8 methylene carbons to be incorporated into the lower layer and 4 water molecules per porphyrin molecule in the upper layer. Calculations also showed that there was a penetration of 11.2 water molecules per porphyrin molecule incorporated into the lower layer at this slightly higher surface pressure.

$\mathrm{X}$-ray reflectometry measurements were also performed at the higher surface pressure of $42 \mathrm{mN} \mathrm{m}^{-1}$, in the second condensed phase observed in the surface pressure-area isotherm (arrow 4 in Figure 2). The best fit to the data was a four-layer model, which is shown in the inset to Figure 3c). This is equivalent to a trilayer at the interface, composed of the monolayer over a bilayer consisting of the interdigitated combination of the monolayers after folding or buckling (see Figure 6).

BAM Images. BAM images were obtained throughout the range of surface pressure shown in Figure 2, although it was difficult to obtain clear images before the pressure peak as there was considerable movement of the structures we observed. At liftoff in Figure 4a, the image shows a mixture of round and stringy domains on a darker background, which is recognized as representative of the liquid gaseous/liquid expanded regime of two phases in coexistence. There are large darker areas with nothing on the surface (dark domain in lower left of Figure 4a) as expected at such a low surface pressure.

Figure $4 b$, taken in the middle of the first region of steeply rising surface pressure of the isotherm, shows an image of a textured surface of islands of material on the surface. In Figure $4 \mathrm{c}$, the movement of these islands of material accounts for the a)

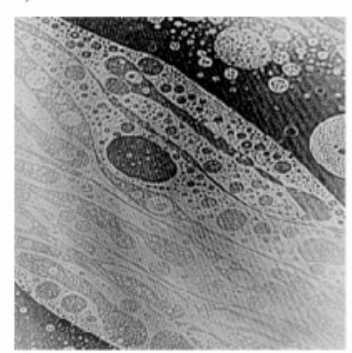

e)

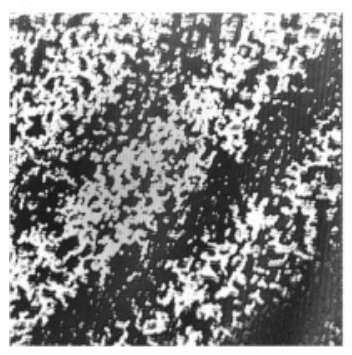

b)

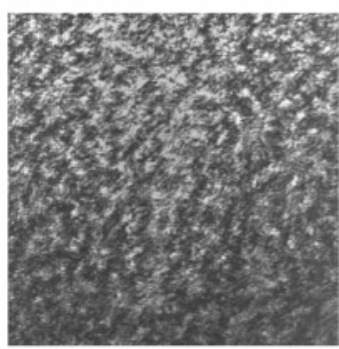

d)

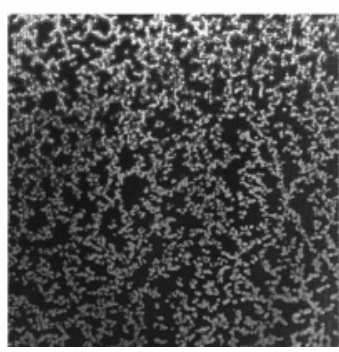

c)

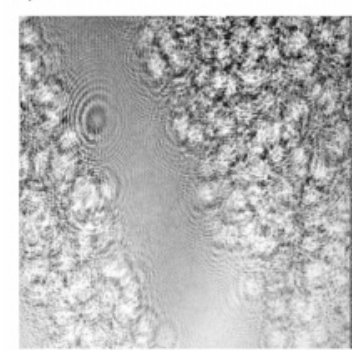

f)

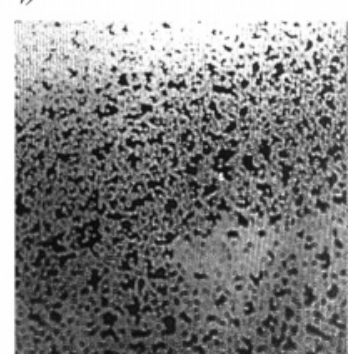

Figure 4. BAM images of the monolayer at the air-water interface formed from the $4: 1$ DHDABr-TSPP complex. (a) $5 \mathrm{mN} \mathrm{m}^{-1}, 140 \AA^{2}$ molec $^{-1}$; (b) $18 \mathrm{mN} \mathrm{m}^{-1}, 100 \AA^{2} \mathrm{molec}^{-1}$; (c) $30 \mathrm{mN} \mathrm{m}^{-1}, 84 \AA^{2} \mathrm{molec}^{-1}$; (d) $34.5 \mathrm{mN} \mathrm{m}^{-1}, 70 \AA^{2} \mathrm{molec}^{-1}$; (e) $34.5 \mathrm{mN}^{-1} \mathrm{~m}^{-1}, 40 \AA^{2} \mathrm{molec}^{-1}$; (f) $42 \mathrm{mN} \mathrm{m}^{-1}, 27 \AA^{2} \mathrm{molec}^{-1}$. 


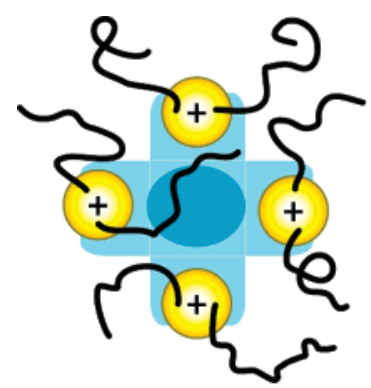

a) Top view

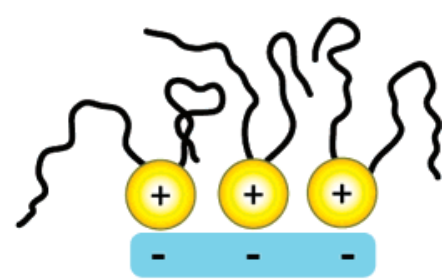

b) Side view
Figure 5. Top and side views of the compressed surfactant-porphyrin complex showing the proposed packing arrangements and alkyl chain conformation. Note that the electrostatic interactions prevent close packing of the alkyl chains and that the disordered chains contribute to the high area per porphyrin.

slightly blurred image obtained. Figure $4 d$, taken at the beginning of the plateau region where the X-ray and neutron data were measured, shows a series of ridges of lighter shaded material in among a darker matrix. We believe that these are signs of the buckling and that the lighter regions are the raised regions where a fold has collapsed back onto the monolayer as shown in Figure 6. The movement of the islands of material has ceased, indicating that there is an interconnected coverage of the surface. In Figure $4 \mathrm{e}$, we observe an increase in the coverage of the ridges. The appearance of the ridges is caused by localized folding, estimated to be occurring on the micron scale from closer examination of the images $4 d-f$. In Figure $4 \mathrm{f}$, the lighter areas have increased in density so that it appears that the surface is almost completely covered by a trilayer.

\section{Discussion}

Perez-Morales et al. ${ }^{19}$ examined a similar system of a 4:1 molar mixture of a dioctadecyldimethylammonium bromide and TSPP complex at the air-water interface using surface pressure-area isotherms, UV-vis spectroscopy, and circular dichroism. Their system showed a surface pressure-area isotherm similar to the 4:1 TSPP-DHDABr complex studied in this work. The authors used their observations of measurements from the monolayer to infer that the pressure peak was the result of a porphyrin monomer-dimer transition. An additional Soret band was observed in the UV-vis spectra at lower wavelength, which was also attributed to the formation of the dimer.

The first of the three zones identified in the isotherm of the complex begins when the area per DHDABr molecule reaches
$150 \AA,^{2}$ which is equivalent to an area of $600 \AA^{2}$ per porphyrin molecule. At such large molecular areas, the complex exists as a loose arrangement of islands of material as we observe in Figure 4a. At the surface pressure peak, the equivalent area per porphyrin measured from the isotherm has reduced to $304 \AA{ }^{2}$ It has been established that even with bulky functional groups attached at the meso position the porphyrin takes up a maximum area of approximately $320 \AA^{2}$ at the interface in a parallel orientation. ${ }^{9}$ BAM images taken throughout the first region (Figure $4 \mathrm{a}-\mathrm{c}$ ) are blurred due to movement of domains on the surface, and there were dark regions observed between domains indicating areas of no monolayer coverage. In contrast, images taken after further compression of the monolayer beyond the surface pressure peak (Figure $4 \mathrm{~d}-\mathrm{f}$ ) show no movement, and the surface coverage appears to be complete. At the end of the first region of sharply increasing surface pressure we suggest that the complex has formed a complete monolayer. The further reduction of molecular area observed through the plateau region results from a gradual process of collapse toward the trilayer structure.

Models obtained from simultaneous fitting of X-ray and neutron isotopically contrasted data measured on either side of the pressure peak reveal a surface complex composed of two layers of almost equal thickness. The upper layer, which is assumed to be composed of the hydrophobic alkyl chains, is thinner than predicted for alkyl chains of 16 carbon atoms. The alkyl chains are often able to form a close-packed but thinner layer due either to some tilt of the chains away from the surface normal or gauche defects of the chains. In our experiment, the alkyl chains are unable to form a close-packed array (see Figure 5) as the electrostatic interactions between the surfactant headgroups and sulfonato groups on the porphyrin constrain the proximity of the surfactant headgroups. This less compressed arrangement results in more gauche defects and a thinner and less dense alkyl chain layer, as was observed.

There is a reduction in molecular area from the beginning of the plateau region to the second condensed phase from 76 to $29 \AA^{2}$ per DHDABr molecule. Modeling reflectometry data have revealed that there were no significant structural changes to the two layers of the complex before and after the pressure peak. In Figure $4 d-f$, taken after the pressure peak, there is evidence of ridges resulting from folding, and the focused images indicate that the movement had ceased. A gradual increase in surface coverage was also observed. Hence, the reduction in molecular area is achieved through a gradual process of local folding or buckling of the rigid complex to form areas of trilayer coverage

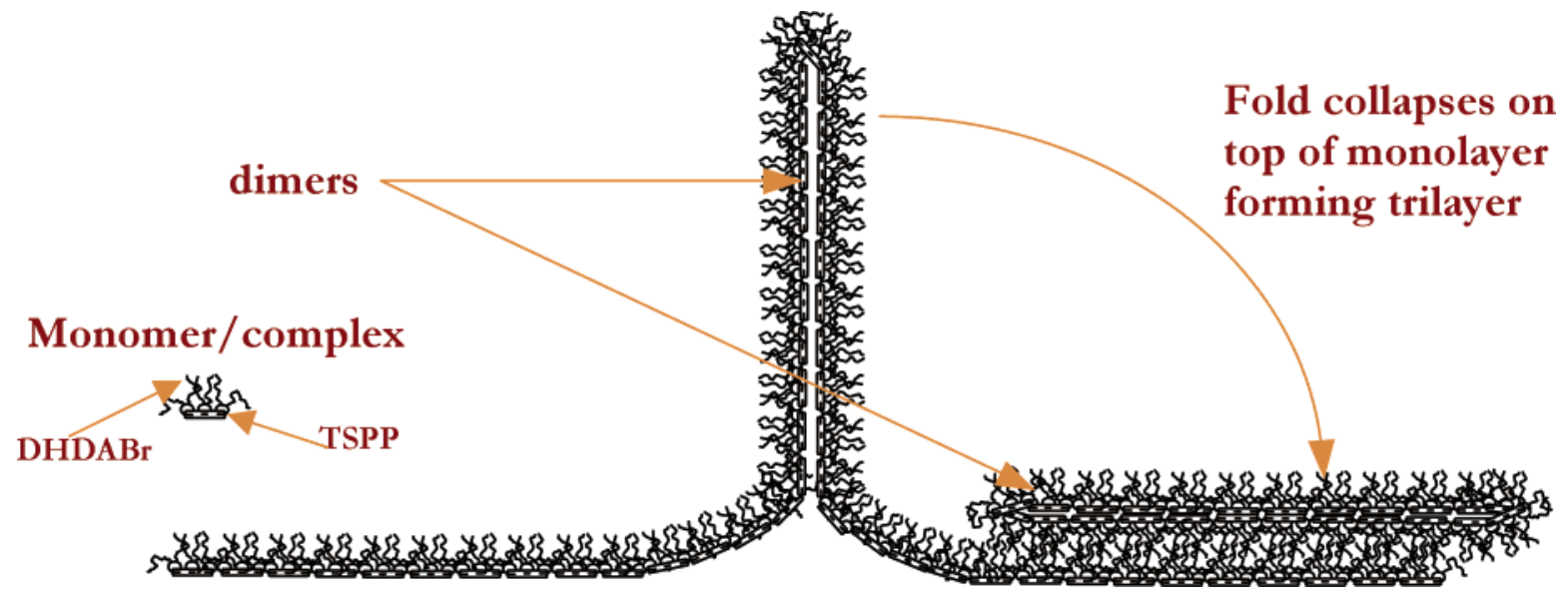

Figure 6. Folding of complex at high surface pressure; arrows show possible porphyrin dimers formation due to the bending or buckling of the monolayer. 
as depicted in Figure 6. The maximum or surface pressure peak represents an energetic barrier to the beginning of the buckling or folding process. Together, the evidence supports the idea that the surface pressure peak represents the change from an amalgamation process to one of folding and buckling.

The large molecular area of the $4: 1$ porphyrin complex of $304 \AA^{2}$ indicates that the surfactant molecules coordinated to the sulfonato groups at the meso position add to the effective molecular area of the complex. The constrained simultaneous modeling of neutron and X-ray data shows that there is a considerable amount of tail material included in the headgroup layer. In Figure 5, the top view of the complex shows how it is likely that the monolayer is formed by the interactions of alkyl chains from adjacent porphyrins. Such an arrangement has been observed previously for alkylated porphyrins adsorbed on a graphite basal plane surface. ${ }^{28,29}$

The results we have obtained indicate that the purported monomer-dimer transition observed by other researchers corresponds to the change from monolayer to trilayer through film folding occurring throughout the plateau region. The presence of dimers or stacked porphyrins occurs as the monolayer folds to form an interdigitated bilayer at the interface with another monolayer on top (see Figure 6). The final condensed phase begins at an area per surfactant molecule of $29 \AA,{ }^{2}$ which in terms of a trilayer coverage is equivalent to $29 \AA^{2} \times 3$ (trilayer) $\times 4$ (per porphyrin) $=348 \AA{ }^{2}$ which based on the area per molecule of $304 \AA^{2}$ is equivalent to a coverage of the trilayer of $88 \%$. It is obvious from the mottled appearance in the BAM images from this region of the isotherm that the coverage is less than $100 \%$.

The third phase observed in the surface pressure-area isotherms represents the buckled trilayer arrangement. The fitted reflectometry data taken from the beginning of the final condensed phase in Figure $3 \mathrm{c}$ show a trilayer that consists of an interdigitated bilayer lying at the interface with another monolayer above as shown in Figure 6. Such a collapsed trilayer has been observed before for amphiphilic monolayers at the interfaces as a result of local folding and bending. ${ }^{22}$

\section{Conclusion}

The surfactant DHDABr and the porphyrin TSPP form an electrically neutral complex in the molar ratio of $4: 1$, respectively. The surface pressure isotherm of the complex is different from the isotherm of the pure surfactant and has three distinct regions: an initial condensed phase, an interim plateau which follows a surface pressure peak, and a final condensed phase. Constrained simultaneous fitting of two neutron contrasts and $\mathrm{X}$-ray reflectometry data revealed that a significant proportion of the alkyl chains exists in the headgroup region. Modeling $\mathrm{X}$-ray reflectometry data also confirm that in the final condensed phase there is an almost complete surface coverage of a trilayer formed by buckling and collapse of a bilayer of the film on top of the monolayer. BAM images of the complex before the surface pressure peak reveal that the initial condensed phase observed in the isotherm represents formation of complete surface coverage. BAM also reveals that after the peak the process of trilayer formation occurs in the plateau region as the film buckles and folds.

Acknowledgment. The authors thank Prof. R. K. Thomas for the kind donation of a sample of deuterated DHDABr. Travel grants through the Australian Government Access to Major Research Facilities Program are gratefully acknowledged, as is ARC funding to facilitate access to ISIS. The reflectometry experiments were performed at the Advanced Photon Source with support from the Australian Synchrotron Research Program, which is funded by the Commonwealth of Australia under the Major National Research Facilities Program. Use of the Advanced Photon Source was supported by the U.S. Department of Energy, Office of Science, Office of Basic Energy Sciences, under Contract No. W-31-109-Eng-38.

\section{References and Notes}

(1) Chowdhury, A.; Chowdhury, J.; Pal, P.; Pal, A. J. Solid State Commun. 1998, 107, 725.

(2) Arnold, D. P.; Manno, D.; Micocci, G.; Serra, A.; Tepore, A.; Valli, L. Thin Solid Films 1998, 327-329, 341.

(3) Gregory, B. W.; Vaknin, D.; Gray, J. D.; Ocko, B. M.; Cotton, T. M.; Struve, W. S. J. Phys. Chem. B 1999, 103, 502.

(4) Gregory, B. W.; Vaknin, D.; Gray, J. D.; Ocko, B. M.; Stroeve, P.; Cotton, T. M.; Struve, W. S. J. Phys. Chem. 1997, 101, 2006.

(5) Vergelt, F. J.; Koehorst, R. B. M.; Hoek, A. V.; Schaafsma, T. J. J. Phys. Chem. 1995, 99, 4397.

(6) Hambright, P. Chemistry of water soluble porphyrins. In The Porphyrin Handbook; Kadish, K. M., Smith, K. M., Guillard, R., Eds.; Academic Press: New York, 2000; Vol. 3, p 129.

(7) Qian, D.-J.; Nakamura, C.; Miyake, J. Langmuir 2000, 9615.

(8) Pedrosa, J. M.; Perez, M.; Prieto, I.; Matin-Romero, M. T.; Mobius, D.; Camacho, L. Phys. Chem. Chem. Phys. 2002, 4, 2329.

(9) Martin, M. T.; Prieto, I.; Camacho, L.; Mobius, D. Langmuir 1996, $12,6554$.

(10) Prieto, I.; Romero, M. T. M.; Camacho, L. Langmuir 1998, 14 4175 .

(11) Dynarowicz, P.; Romeu, N. V.; Trillo, J. M. Colloids Surf., APhysicochem. Eng. Aspects 1998, 131, 249.

(12) Penfold, J.; Sivia, D. S.; Staples, E.; Tucker, I.; Thomas, R. K. Langmuir 2004, 20, 2265.

(13) Prosser, A. J.; Franses, E. I. J. Colloid Interface Sci. 2001, 240 590.

(14) Goncalves da Silva, A. M.; Romao, R. S.; Costa, S. M. B. Chem. Phys. Lipids 2004, 127, 77.

(15) Zhang, L.; Qing, L.; Minghua, L. J. Phys. Chem. B 2003, 107, 2565.

(16) Penfold, J.; Richardson, R. M.; Zarbakhsh, A.; Webster, J. R. P.; Bucknall, D. G.; Rennie, A. R.; Jones, R. A. L.; Cosgrove, T.; Thomas, R. K.; Higgins, J. S.; Fletcher, P. D. I.; Dickinson, E.; Roser, S. J.; McLure, I. A.; Hillman, A. R.; Richards, R. W.; Staples, E. J.; Burgess, A. N.; Simister, E. A.; White, J. W. J. Chem. Soc., Faraday Trans. 1997, 93, 38993917. 1369.

(17) Penfold, J.; Thomas, R. K. J. Phys.: Condens. Matter 1990, 2 ,

(18) Nelson, A. J. Appl. Crystallogr. 2006, 39, 273.

(19) Perez-Morales, M.; Pedrosa, J. M.; Martin-Romero, M. T.; Mobius, D.; Camacho, L. J. Phys. Chem. B 2004, 108, 4457.

(20) Lu, J. R.; Simister, E. A.; Thomas, R. K.; Penfold, J. J. Phys.: Condens. Matter 1994, 6, A 403

(21) Bell, G. R.; Manning-Benson, S.; Bain, C. D. J. Phys. Chem. B 1998, 102, 218 .

(22) Reis, H. E.; Swift, H. Langmuir 1987, 3, 853.

(23) Brinkhuis, R. H. G.; Schouten, A. J. Macromolecules 1991, 24, 1487.

(24) Saville, P. M.; Gentle, I. R.; White, J. W.; Penfold, J.; Webster, J. R. P. J. Phys. Chem. 1994, 98, 5935.

(25) Martin, S. M.; Ward, M. D. Langmuir 2005, 21, 5324.

(26) Holman, K. T.; Pivovar, A. M.; Ward, M. D. Science 2000, 294, 1907.

(27) Holman, K. T.; Martin, S. M.; Parker, D. P.; Ward, M. D. J. Am. Chem. Soc. 2001, 123, 4421.

(28) Qiu, X.; Wang, C.; Yin, S.; Zeng, Q.; Xu, B.; Bai, C. J. Phys. Chem. B 2000, 104, 3570.

(29) Qui, X.; Wang, C.; Zeng, Q.; Xu, B.; Yin, S.; Wang, H.; Xu, S.; Bai, C. J. Am. Chem. Soc. 2000, 122, 5550 